\title{
SOME PROPERTIES OF THE SOLUTION OF THE RAMSEY MODEL
}

\author{
loana VIAŞU 1
}

DOI: 10.1515/tjeb-2015-0006

\begin{abstract}
The main aim of this paper is to improve some results obtained by Smith. We provide here a closed-form solution for the Ramsey model. We prove that if the inverse of the constant elasticity of intertemporal substitution is different to the elasticity of output with respect to physical capital, then the economy described by this model reaches the unique steady-state equilibrium, for any starting values of per-capita consumption. The advantage of the paper over the existing paper is that the arguments here are not local, that is, our hypotheses are most generally possible. Consequently, we can obtain almost all other results as particular cases of our solution. Our approach clarifies why different starting values of per-capita consumption generates the same steady-state equilibrium, but of different periods of time. We also clarifiy some of previous result obtained by other authors as for example Luca Guerrini.
\end{abstract}

Keywords: $\quad$ Ramsey growth model, Hamiltonian function, uniqueness and indeterminacy.

\section{JEL Classification: C61, C62, 041.}

${ }^{1}$ Lecturer PhD, West University of Timisoara, Romania. 


\section{Introduction}

The growth model developed by Ramsey (1928) and later refined by Cass (1965) and Koopmans (1968), is one of the most famous theoretical models in modern macroeconomics. There is a long list of papers studying this model. Only under some special hypotheses is possible to derive an explicit solution for this model. Among the most important contributions to this field we cite here the papers of Kurz (1968), Barro et al. (1995), Wälde (2005), under the hypothesis of the constant saving rate and Mehlum (2005) who has provided a closed-form solution for the case of a Leontieff technology. Smith (2006) provides an explicit solution using Gaussian hypergeometric functions, under the hypothesis that capital's share is equal to the reciprocal of the intertemporal elasticity of substitution. This hypothesis was also considered by Guerrini (2010) in a list of successive published papers. More recently, Naz, Mahomed and Chaudhry (2014) develop a partial Hamiltonian framework to obtain reductions and closed-form solutions via first integrals of current value Hamiltonian systems of ordinary differential equations.

The starting point of our approach is the paper of Smith (2006), and the main aim of our paper is to improve some of his results. Smith proved that under the hypotheses $\sigma=\beta$ and the constancy of $c / k$, given the initial capital stock $k$ there is a unique value of initial percapita consumption co that will satisfy the transversality condition.

As it is well-known, Cass proves that for any starting value of ko, an initial value co can be chosen in such a way that the path starting from these values and satisfying the optimality conditions asymptotically approaches to the steady-state equilibrium. We prove in our paper more than this. If $\sigma \neq \beta$ and the ratio $c / k$ is not constant, then the economy described by the Ramsey model reaches the unique steady-state equilibrium for any starting value of $c_{0}$, but the economy needs different periods of time to reach this equilibrium. We also prove that, if $\sigma \neq \beta$ and the ratio $c / k$ is constant for all $t \geq 0$, then the system starts at the steady-state equilibrium.

The outline of the paper is as follows. The first section is this introduction. In the second section we give a succinct description of the Ramsey model, in the third section we present our main result and finally some conclusions.

\section{The Model of Ramsey}

We start this section by considering the standard Ramsey model, where there is neither population growth nor technological growth. Without loss of generality, we suppose that the economy is populated by a large and constant number of identical agents, normalized to

DE GRUYTER OPEN 
one, so that all the variables can be interpreted as per capita quantities. The model is characterized by the well-known optimization problem.

Definition 1 The set of paths $\{k(t) ; c(t)\}$ is called an optimal solution if it solves the following optimization problem:

$$
V_{0}=\max _{c(t)} \int_{0}^{\infty} \frac{[c(t)]^{1-\sigma-1}}{1-\sigma} e^{-\rho t} d t
$$

subject to:

$$
\left\{\begin{array}{c}
\dot{k}(t)=\gamma[k(t)]^{\beta}-\pi k(t)-c(t) \\
k_{0}=k(0)
\end{array}\right.
$$

$\beta$ is the elasticity of output with respect to physical capital, $\gamma$ represents an efficiency parameter, $\pi$ is the rate of depreciation, $\rho$ is a positive discount factor, $\sigma^{-1}$ represents the constant elasticity of intertemporal substitution and throughout this paper we suppose that $\sigma \neq \beta . k(t)$ is the physical capital and $c(t)$ is the real consumption.

In this model $\mathrm{c}(\mathrm{t})$ is the control variable and $\mathrm{k}(\mathrm{t})$ is the state variable. The equations (2) give the resources constraints and initial value $k_{0}=k(0)>0$ for the state variables $k(t)$. Of course, the state variable and the control variable are both functions of time, but when no confusions are possible, we simply write $\mathrm{k}$ and $\mathrm{c}$.

To solve the problem (1) subject to (2), we define the Hamiltonian function:

$$
H=\frac{c^{1-\sigma}-1}{1-\sigma}+\left[\gamma k^{\beta}-\pi k-c\right] \lambda \text { - }^{-}
$$

The boundary conditions include the initial value $\mathrm{k}_{0}$ and the transversality condition: $\lim _{t \rightarrow \infty} e^{-\rho t} \lambda(t) k(t)=0$. In an optimal program the control variable is chosen so as to maximize $\mathrm{H}$. We note that along the optimal path, $\lambda$ is a function of $t$ only. The necessary first order conditions for $\mathrm{c}$ to be an optimal control are:

$$
\left\{\begin{array}{l}
\frac{\dot{\mathrm{k}}}{\mathrm{k}}=\gamma \frac{1}{\mathrm{k}^{1-\beta}}-\pi-\frac{\mathrm{c}}{\mathrm{k}} \\
\frac{\dot{\mathrm{c}}}{\mathrm{c}}=\frac{\gamma \beta}{\sigma} \frac{1}{\mathrm{k}^{1-\beta}}-\frac{\rho+\pi}{\sigma}
\end{array},\right.
$$

These equations, together with the two boundary conditions, the initial condition and transversality condition, constitute the dynamic system which drives the economy over time.

\section{DE GRUYTER}

\section{OPEN}

Timisoara Journal of Economics and Business | ISSN: 2286-0991 | www.tjeb.ro

Year 2014 | Volume 7 | Issue 2 | Pages: 113-122 
Viaşu, I. (2014).

Some Properties of the Solution of the Ramsey Model

As it is well-known, the steady-state equilibrium, which is defined as a situation in which the growth rates of consumption and capital are zero, is given by:

$$
\left\{\begin{array}{c}
k_{*}=\left(\frac{\gamma \beta}{\pi+\rho}\right)^{\frac{1}{1-\beta}} \\
\xi=\frac{c_{*}}{k_{*}}=\frac{\pi(1-\beta)+\rho}{\beta}
\end{array}\right.
$$

\section{Uniqueness and Indeterminacy}

In order to proof our main result, we need the following preliminary result.

Lemma 1 Under the hypothesis $\sigma \neq \beta$, for every real $\varepsilon>0$, there exists a finite $t_{*}>0$ such that for all $t>t_{*}$,

$$
\left[\frac{c(t)}{k(t)}-\xi\right]<\epsilon
$$

Theorem 1 Under the hypothesis $\sigma \neq \beta$, the system (3) has the following solution

$$
\begin{gathered}
k(t)=e^{-[\pi t+\widetilde{D}(t)]}\left[\left(e^{\widetilde{D}_{0}} k_{0}\right)^{1-\beta}+\gamma(1-\beta) \int_{0}^{t} g(s) d s\right]^{\frac{1}{1-\beta}} \\
c(t)=c_{0}\left[e^{\widetilde{D}(t)} k_{0}\right]^{-\frac{\beta}{\sigma}}\left[\left(e^{\widetilde{D}_{0}} k_{0}\right)^{1-\beta}+\gamma(1-\beta) \int_{0}^{t} g(s) d s\right]^{\frac{\beta}{\sigma(1-\beta)}} e^{-\frac{\rho+\pi}{\sigma} t}
\end{gathered}
$$

that is asymptotically convergent to the unique steady-state equilibrium, for any starting value of $\mathrm{c}_{0}$, where

$$
g(t)=e^{(1-\beta)[\pi t+\widetilde{D}(t)]},
$$

$$
\dot{D}(t)=(1-\beta)[\pi+a(t)], \Rightarrow D(t)=(1-\beta)[\pi t+\widetilde{D}(t)]
$$

With

$$
\widetilde{D}(t)=\widetilde{D_{0}}+\int_{0}^{t} a(s) d s, \quad a(t)=\frac{c(t)}{k(t)} \text { and } \widetilde{D_{0}}=\widetilde{D}(0)
$$


Viaşu, I. (2014).

Some Properties of the Solution of the Ramsey Model

Proof of Theorem 1. Let us consider the first equation of the system (3)

$$
\frac{\dot{k}}{k}=\gamma \frac{1}{k^{1-\beta}}-\pi-\frac{c}{k}
$$

In order to solve the system, we denote by $x=k^{1-\beta}$ to obtain the following linear differential equation:

$$
\dot{x}=-(1-\beta)(\pi+a) x+\gamma(1-\beta)
$$

whose solution is given by

$$
x(t)=e^{-D(t)}\left[e^{D_{0}} x_{0}+\gamma(1-\beta) \int_{0}^{t} e^{(1-\beta)[\pi t+\widetilde{D}(t)]} d s\right], x_{0}=x(0) .
$$

Under the above notations we obtain the following solution for $\mathrm{k}$.

$$
k(t)=e^{-[\pi t+\widetilde{D}(t)]}\left[\left(e^{\widetilde{D}_{0}} k_{0}\right)^{1-\beta}+\gamma(1-\beta) \int_{0}^{t} g(s) d s\right]^{\frac{1}{1-\beta}}
$$

Substituting this result into the second equation of the system (3) we obtain the following differential equation

$$
\frac{\dot{c}}{c}=\frac{\gamma \beta}{\sigma} \frac{e^{(1-\beta)[\pi t+\widetilde{D}(t)]}}{\left(e^{\widetilde{D}_{0}} k_{0}\right)^{1-\beta}+\gamma(1-\beta) \int_{0}^{t} e^{(1-\beta)[\pi t+\widetilde{D}(t)]} d s}-\frac{\rho+\pi}{\sigma} .
$$

Observe now that the function:

$$
G(t)=\left(e^{\widetilde{D}_{0}} k_{0}\right)^{1-\beta}+\gamma(1-\beta) \int_{0}^{t} e^{(1-\beta)[\pi t+\widetilde{D}(t)]} d s
$$

has the following property: $\dot{G}(t)=\gamma(1-\beta) g(t)$ and therefore we can write

$$
\frac{\dot{c}}{c}=\frac{\beta}{\sigma(1-\beta)} \frac{\dot{G}}{G}-\frac{\rho+\pi}{\sigma}
$$

and the solution is given by

$$
\ln \left[\frac{c(t)}{c_{0}}\right]=\frac{\beta}{\sigma(1-\beta)} \ln \left[\frac{G(t)}{G_{0}}\right]+\ln \left[e^{-\frac{\rho+\pi}{\sigma} t}\right]
$$


Viaşu, I. (2014).

Some Properties of the Solution of the Ramsey Model

$$
c(t)=c_{0}\left[e^{\widetilde{D}_{0}} k_{0}\right]^{-\frac{\beta}{\sigma}}\left[\left(e^{\widetilde{D}_{0}} k_{0}\right)^{1-\beta}+\gamma(1-\beta) \int_{0}^{t} g(s) d s\right]^{\frac{\beta}{\sigma(1-\beta)}} e^{-\frac{\rho+\pi}{\sigma} t}
$$

In order to prove the transversality condition, using the Lemma 1 we can write

$$
\widetilde{D}(t)=\widetilde{D_{0}}+\widetilde{D_{*}}+\xi\left(t-t_{*}\right), \quad \text { where } \widetilde{D_{*}}=\int_{0}^{t_{*}} a(s) d s
$$

and thus, the transversality condition is satisfied for any starting value of $c_{0}$. Indeed we have:

$$
\begin{gathered}
\lim _{t \rightarrow \infty} k(t)[c(t)]^{-\sigma} e^{-\rho t}=\frac{\left[e^{\widetilde{D}_{0}} k_{0}\right]^{\beta}}{c_{0}^{\sigma}} \lim _{t \rightarrow \infty} \frac{\left(e^{\widetilde{D}_{0}} k_{0}\right)^{1-\beta}+\gamma(1-\beta) \int_{0}^{t} g(s) d s}{e^{\widetilde{D}(t)}}= \\
=\frac{\left[e^{\widetilde{D}_{0}} k_{0}\right]^{\beta}}{c_{0}^{\sigma}} \lim _{t \rightarrow \infty} \frac{\gamma(1-\beta) e^{(1-\beta)[\pi t+\widetilde{D}(t)]}}{\xi e^{\widetilde{D}(t)}} \\
=\frac{\left[e^{\widetilde{D}_{0}} k_{0}\right]^{\beta}}{c_{0}^{\sigma}} \frac{\gamma \beta(1-\beta)}{\rho+\pi(1-\beta)} \lim _{t \rightarrow \infty} e^{\pi(1-\beta) t-\beta \widetilde{D}(t)} \\
=\frac{\left[e^{\widetilde{D}_{0}} k_{0}\right]^{\beta}}{c_{0}^{\sigma}} \frac{\gamma \beta(1-\beta)}{\rho+\pi(1-\beta)} \lim _{t \rightarrow \infty} e^{-\rho t-\beta\left[\widetilde{D_{0}}+\widetilde{D_{*}}-\xi t_{*}\right]}=0 .
\end{gathered}
$$

What we need now is to prove that

$$
\begin{gathered}
\lim _{t \rightarrow \infty}[k(t)]^{1-\beta}=k_{*}^{1-\beta} \text { and } \lim _{t \rightarrow \infty} c(t)=\frac{\pi(1-\beta)+\rho}{\beta} k_{*} . \\
\lim _{t \rightarrow \infty} e^{-(1-\beta)[\pi t+\widetilde{D}(t)]}\left[\left(e^{\widetilde{D}_{0}} k_{0}\right)^{1-\beta}+\gamma(1-\beta) \int_{0}^{t} e^{(1-\beta)[\pi t+\widetilde{D}(t)]} d s\right] \\
\lim _{t \rightarrow \infty} \frac{\left(e^{\widetilde{D}_{0}} k_{0}\right)^{1-\beta}+\gamma(1-\beta) \int_{0}^{t} e^{(1-\beta)[\pi t+\widetilde{D}(t)]} d s}{e^{(1-\beta)[\pi t+\widetilde{D}(t)]}}=\frac{\gamma \beta}{\pi+\rho}=k_{*}^{1-\beta} . \\
\lim _{t \rightarrow \infty} \frac{\left(e^{\widetilde{D}_{0}} k_{0}\right)^{1-\beta}+\gamma(1-\beta) \int_{0}^{t} g(s) d s}{e^{\frac{(1-\beta)(\rho+\pi)}{\beta} t}}=\frac{\gamma \beta}{\rho+\pi} \lim _{t \rightarrow \infty} \frac{e^{(1-\beta)[\pi t+\widetilde{D}(t)]}}{e^{\frac{(1-\beta)(\rho+\pi)}{\beta} t}}
\end{gathered}
$$


Viaşu, I. (2014).

Some Properties of the Solution of the Ramsey Model

$$
=\frac{\gamma \beta}{\rho+\pi} \lim _{t \rightarrow \infty} e^{(1-\beta)\left[\pi t-\frac{\rho+\pi}{\beta} t+\widetilde{D}(t)\right]}=\frac{\gamma \beta}{\rho+\pi} e^{(1-\beta)\left(\widetilde{D}_{0}+\widetilde{D}_{*}-\xi t_{*}\right)}
$$

and therefore we have

$$
\begin{aligned}
& \lim _{t \rightarrow \infty} c(t)=c_{0}\left[e^{\widetilde{D}_{0}} k_{0}\right]^{-\frac{\beta}{\sigma}}\left[\frac{\gamma \beta}{\rho+\pi} e^{(1-\beta)\left(\widetilde{D}_{0}+\widetilde{D}_{*}-\xi t_{*}\right)}\right]^{\frac{\beta}{\sigma(1-\beta)}} \\
& =\frac{c_{0}}{k_{0}^{\frac{\beta}{\sigma}}}\left[\frac{\gamma \beta}{\rho+\pi}\right]^{\frac{\beta}{\sigma(1-\beta)}} e^{\beta \frac{\left(\widetilde{D}_{*}-\xi t_{*}\right)}{\sigma}}=c_{0} \frac{k_{*}^{\frac{\beta}{\sigma}}}{k_{0}^{\frac{\beta}{\sigma}}} e^{\beta \frac{\left(\widetilde{D}_{*}-\xi t_{*}\right)}{\sigma}}
\end{aligned}
$$

and this limit equals $\xi k_{*}$ only if

$$
c_{0}=\xi k_{0}^{\frac{\beta}{\sigma}} k_{*}^{\frac{\sigma-\beta}{\sigma}} e^{\frac{\beta\left(\xi t_{*}-\widetilde{D}_{*}\right)}{\sigma}} \Rightarrow \frac{c_{0}}{k_{0}}=\frac{k_{*}^{\frac{\sigma-\beta}{\sigma}}}{k_{0 *}^{\frac{\sigma-\beta}{\sigma}}} e^{\frac{\beta\left(\xi t_{*}-\widetilde{D}_{*}\right)}{\sigma}}
$$

Now it is clear that the system reaches the unique steady-state equilibrium for any starting point $c_{0}$. As we can see from the above relation, for different starting values of co, the system reaches the same steady-state equilibrium, but it needs different periods of time, and thus the proof is completed.

The next proposition gives our second result and tries to shed some light on the results obtained by Smith.

Proposition 1 Let us consider the system (3) which drives the economy over time.

1. If $\sigma \neq \beta$ and $\mathrm{c}(\mathrm{t}) / \mathrm{k}(\mathrm{t})=\xi$ for all $\mathrm{t} \geq 0$, then the system (3) has the following solution:

$$
k(t)=k_{*} \text { and } c(t)=c_{*}
$$

2. If $\sigma=\beta$ and $\mathrm{c}(\mathrm{t}) / \mathrm{k}(\mathrm{t})=\xi$ for all all $\mathrm{t} \geq 0$, then the system (3) has the Smith solution.

Proof of Proposition 1. If $\mathrm{c}(\mathrm{t}) / \mathrm{k}(\mathrm{t})=\xi$ for all $\mathrm{t} \geq 0$, then $\widetilde{D}(t)=\xi t$ and $\widetilde{D}(0)=0$ and consequently, the solutions (5) and (6) can therefore be written 
Viaşu, I. (2014).

Some Properties of the Solution of the Ramsey Model

$$
\begin{gathered}
\begin{aligned}
& k(t)=e^{-(\pi+\xi) t}\left[k_{0}^{1-\beta}+\gamma(1-\beta) \int_{0}^{t} e^{(1-\beta)(\pi+\xi) s} d s\right]^{\frac{1}{1-\beta}} \\
&=\left[\left(k_{0}^{1-\beta}+\frac{\gamma \beta}{\pi+\rho}\right) e^{-\frac{(\pi+\rho)(1-\beta)}{\beta} t}+\frac{\gamma \beta}{\pi+\rho}\right]^{\frac{1}{1-\beta}} \\
& c(t)=c_{0} k_{0}^{-\frac{\beta}{\sigma}}\left[k_{0}^{1-\beta}+\gamma(1-\beta) \int_{0}^{t} e^{(1-\beta)(\pi+\xi) s} d s\right]^{\frac{\beta}{\sigma(1-\beta)}} e^{-\frac{\rho+\pi}{\sigma} t} \\
&=c_{0} k_{0}^{-\frac{\beta}{\sigma}}\left[\left(k_{0}^{1-\beta}+\frac{\gamma \beta}{\rho+\pi}\right) e^{-\frac{(\pi+\rho)(1-\beta)}{\beta} t}+\frac{\gamma \beta}{\rho+\pi}\right]^{\frac{\beta}{\sigma(1-\beta)}} .
\end{aligned}
\end{gathered}
$$

The transversality condition for these solutions is satisfied for any starting value of co. Indeed we have:

$$
\lim _{t \rightarrow \infty} k(t)[c(t)]^{-\sigma} e^{-\rho t}=\frac{k_{0}^{\beta}}{c_{0}^{\sigma}} \lim _{t \rightarrow \infty}\left[\left(k_{0}^{1-\beta}-\frac{\gamma \beta}{\rho+\pi}\right) e^{-\varphi t}+\frac{\gamma \beta}{\rho+\pi}\right] e^{-\rho t}=0 .
$$

What we need now is to prove that

$$
\lim _{t \rightarrow \infty}[k(t)]^{1-\beta}=k_{*}^{1-\beta} \text { and } \lim _{t \rightarrow \infty} c(t)=\frac{\pi(1-\beta)+\rho}{\beta} k_{*}
$$

The first assertion is obviously true. To prove the second assertion we write:

$$
\lim _{t \rightarrow \infty} c(t)=c_{0} k_{0}^{-\frac{\beta}{\sigma}} \lim _{t \rightarrow \infty}\left[\left(k_{0}^{1-\beta}-\frac{\gamma \beta}{\rho+\pi}\right) e^{-\frac{(\rho+\pi)(1-\beta)}{\beta} t}+\frac{\gamma \beta}{\rho+\pi}\right]^{\frac{\beta}{\sigma(1-\beta)}}
$$

and this yields

$$
c_{*}=c_{0} k_{0}^{-\frac{\beta}{\sigma}} k_{*}^{\frac{\beta}{\sigma}} \Rightarrow \frac{c_{*}}{k_{*}}=\frac{c_{0}}{k_{0}} \frac{\frac{\sigma-\beta}{\frac{\sigma-\beta}{\sigma}}}{k_{*}^{\frac{\sigma-\beta}{\sigma}}}
$$

and because $\sigma \neq \beta$ it follows that $k_{0}=k_{*}$ and consequently $c_{0}=c_{*}$.

Let us now consider the second case. In order to better understand some of the results presented below, when examining the equations developed by Smith, we use his notations. 
Viaşu, I. (2014).

Under the hypothesis $\sigma=\alpha$, Smith obtained, via hypergeometric functions, the following solution

$$
k(t)=\left[\left(k_{0}^{1-\alpha}-\frac{\alpha}{\delta+\rho}\right) e^{-\frac{(\delta+\rho)(1-\alpha)}{\alpha} t}+\frac{\alpha}{\delta+\rho}\right]^{\frac{1}{1-\alpha}}, \frac{c}{k}=\bar{x}=\frac{\rho+\delta(1-\alpha)}{\alpha}
$$

We prove here that the use of these functions is not necessary to obtain this solution. Let us start with equations (11) and (12) presented at page (5) of Smith paper. As he proved in the appendix, by using the hypergeometric functions, the constant of integration A must be equal to zero. In fact, this condition can be immediately obtained from equation (11). The solution $\mathrm{x}(\mathrm{t})$ presented in equation (11) is an admissible solution if and only if $\lim _{t \rightarrow \infty} x(t)=\bar{x}$. Obviously, this is true only if $A=0$. Substituting this result into equation (12) we get

$$
z=-\frac{(\delta+\rho)(1-\alpha)}{\alpha} z+(1-\alpha)
$$

whose solution is given by ( 8 ) and thus the proof is completed.

\section{Conclusions}

The main aim of this paper was to improve some results obtained by Smith. We provide a closed-form solution for the Ramsey model and prove that a unique steady-state equilibrium always exists, under the hypotheses that $\sigma \neq \beta$ and the non-constancy of the ratio c/k. Our approach also clarifies why different starting values of per-capita consumption generates the same steady-state equilibrium, but of different periods of time. Our approach also clarifies some of previous result obtained by other authors as for example Luca Guerrini. Of course, further research is necessary in order to better understand the dynamics of Ramsey model, especially along the transitional period.

\section{Acknowledgement:}

This work was financially supported through the project This work was financially supported through the project "Routes of academic excellence in doctoral and post-doctoral research-READ" co-financed through the European Social Fund, by Sectoral Operational Programme Human Resources Development 2007-2013, contract no POSDRU /159/1.5/S/137926.

\section{DE GRUYTER} OPEN 
Viaşu, I. (2014).

\section{References}

Barro, R. N., Mankiw ,G., \& Sala-i-Martin, X. (1995). Capital Mobility in Neoclassical Models of Growth. American Economic Review, 85, 103-115.

Cass, D. (1965). Optimum Growth in an Aggregative Model of Capital Accumulation. The Review of Economic Studies, 32(3), 233 - 240. doi:10.2307/2295827

Guerrini, L. (2010a). The Ramsey model with a bounded population growth rate. Journal of Macroeconomics, 32(3), 872-878. doi:10.1016/j.jmacro.2009.08.004

Guerrini, L. (2010b). The Ramsey model with AK technology and a bounded population growth rate. Journal of Macroeconomics, 32(4), 1178-1183. doi:10.1016/j.jmacro.2010.05.001

Guerrini, L. (2010c). A closed-form solution to the Ramsey model with logistic population growth. Economic Modelling, 27(5), 1178-1182. doi:10.1016/j.econmod.2010.03.002

Koopmans, T. (1968). On the Concept of Economic Development. The Economic Approach to Development Planning. Amsterdam, Elsevier.

Kurz, M. (1968). The General Instability of a Class of Competitive Growth Models. Review of Economic Studies, 35, 155 - 174.

Mehlum ,H. (2005). A closed form Ramsey saddle path. The B.E. Journal of Macroeconomics (Contributions), 5 (1), Article 2. doi:10.2202/1534-6005.1267

Naz, R., Mahomed, F. M., \& Chaudhry, A. (2014). A partial Hamiltonian approach for current value Hamiltonian systems. Communications in Nonlinear Science and Numerical Simulations, 19(10), 3600-3610. doi:10.1016/j.cnsns.2014.03.023

Ramsey, F. P., (1928). A mathematical theory of savings. Economic Journal, 38, 543 - 559.

Smith, W. T., (2006). A closed form solution to the Ramsey model. Contributions in Macroeconomics, 6(1), 1-27. doi:10.2202/1534-6005.1356

DE GRUYTER OPEN 